\title{
Fast Registration of Remotely Sensed Images for Earthquake Damage Estimation
}

\author{
Arash Abadpour, ${ }^{1}$ Shohreh Kasaei, ${ }^{2}$ and S. Mohsen Amiri ${ }^{2}$ \\ ${ }^{1}$ Department of Mathematical Science, Sharif University of Technology, P.O. Box 11365-9415, Tehran, Iran \\ ${ }^{2}$ Department of Computer Engineering, Sharif University of Technology, P.O. Box 11365-9517, Tehran, Iran
}

Received 13 February 2005; Revised 16 September 2005; Accepted 26 September 2005

Recommended for Publication by Stephen Marshall

Analysis of the multispectral remotely sensed images of the areas destroyed by an earthquake is proved to be a helpful tool for destruction assessments. The performance of such methods is highly dependant on the preprocess that registers the two shots before and after an event. In this paper, we propose a new fast and reliable change detection method for remotely sensed images and analyze its performance. The experimental results show the efficiency of the proposed algorithm.

Copyright ( 2006 Hindawi Publishing Corporation. All rights reserved.

\section{INTRODUCTION}

In recent years, the spatial and spectral resolutions of remotely sensed sensors and the revisiting frequency of satellites have increased extensively. These developments have offered the possibility of addressing new applications of remote sensing in environmental monitoring. On the other hand, the officials are getting more and more aware of using multispectral remotely sensed images for regular and efficient control of the environment $[1,2]$.

Change detection of remotely sensed images can be viewed as a general case of a global motion estimation usually used in the video coding applications. However, the following should be noted.

(i) In video coding applications, objects are likely to be presented in the next frame unless we have occlusions, newly appeared objects, or lightning changes, or when we deal with degraded images. But, in remote sensing applications for situations such as earthquake, we are faced with very severe situations in which large areas are likely to be totally destroyed.

(ii) In video coding applications, the temporal rate is about 30 frames per second, and thus one can benefit from the existing high temporal redundancy between successive frames (when there is no shot change), while in remote sensing applications, the time interval between two captured multiband images can be considerably long resulting in a very low temporal redundancy.

(iii) In video coding applications, the segmentation and motion estimation stages can in done in a crisp fashion, while in remote sensing applications because of the different range of changes that might exist between two shots, the decisions should be made in a fuzzy fashion to take advantage of its membership style soft decisions.

(iv) In remote sensing applications, the size and the number of the multispectral images are much higher than those in video sequences; and thus even after dimension reduction processes, we still need to have very fast algorithms.

(v) In remote sensing applications, due to the geometrical changes in image capturing conditions, sensor-type changes, and the long interval among captured images, an accurate registration process is required that plays an important role in the overall performance of any change detection or classification algorithm.

According to the above-mentioned problems, the global video motion techniques might be inefficient when dealing with change detection of remote sensing applications. However, the global video motion estimation can be viewed as a special case of the proposed change detection algorithm; and thus the proposed algorithm can be used for such applications as well.

A key issue in analyzing the remotely sensed images is to detect changes on the earth's surface in order to manage possible interventions to avoid massive environmental problems [3]. Recently, many researchers have worked on using the remote-sensing data to help estimate the earthquake's damages $[4,5]$ or the afterwards reconstruction progresses [6]. Change detection algorithms usually take two sets of images as the two ensembles before and after the change, and return 
the locations where the changes are likely to happen [1]. Before such a stage, a preprocessing step is necessary to produce two comparable images.

The process of registration aims at performing some geometrical operations on one of the images (or both of them) to give two compatible images in which the pixels with the same coordinates in the two images correspond to the same physical point [7]. Many researchers have reported the impact of misregistration on the change detection results (e.g., see [8]). The registration operation is an inverse problem trying to compensate the real transformation produced by the imaging conditions. Although different registration methods are introduced and analyzed [7, 9], there is no optimal solution found yet and the problem is still an active research area [10].

The majority of registration methods consist of four essential steps [9]:

(i) feature detection,

(ii) feature matching,

(iii) transfer model estimation,

(iv) image resampling and transformation.

The first step along with the second step aims at finding two sets of corresponding points in the two images. These two sets are used in the second step to estimate the transform model. Finally, the fourth step results in the two registered images.

There are two typical methods for finding and matching feature points. The first one is to search for robust points in the two images. There are reports of using contours [11], boundaries [12], water reservoirs [13, 14], buildings [15], urban areas [16], roads [17], forests [18], coastal line [19], and the forth as the features. Another approach is to use the information theory tools like mutual information to find the control points [20]. All of the above-mentioned approaches perform both feature detection and feature matching at the same time. Due to the massive effect of mismatching of the control points on the final registration results [8], we emphasize on the determination procedure of the assigned control points (even by using the old-style approach of human intervention) for finding a set of about 20 correct control points in the two images. The challenge of using the robust control points is more clear when investigating the postearthquake images (see Figure 1). Note that even if we do not find the related control points in the second image, it still barriers valuable information about the level of occurred changes. It must be emphasized that any automatic control point detection method can be integrated to the proposed method.

Figures 2 and 3 show the used logo image and the different transforms applied on it, respectively. Figure 4 shows the logo image with a set of control points overlaid on it. Figure 5 shows the result of performing our estimated affine transform on the transferred images shown in Figure 3. Here, we have used a new visualization method in which we have put the two registered images in the red-and green-color channels of an image and have filled the blue-color channel with a value of 255. As such, the magenta and cyan pixels will clearly show the misregistered locations. Note that doing as such,

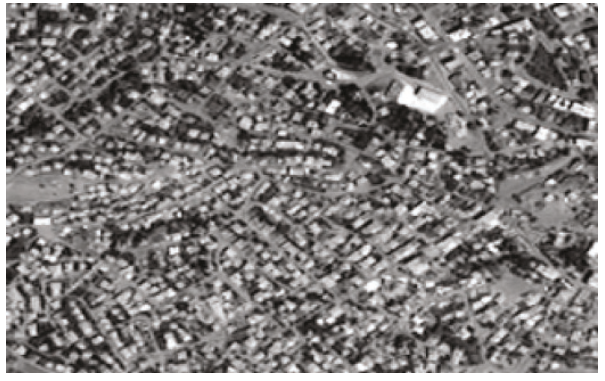

(a)

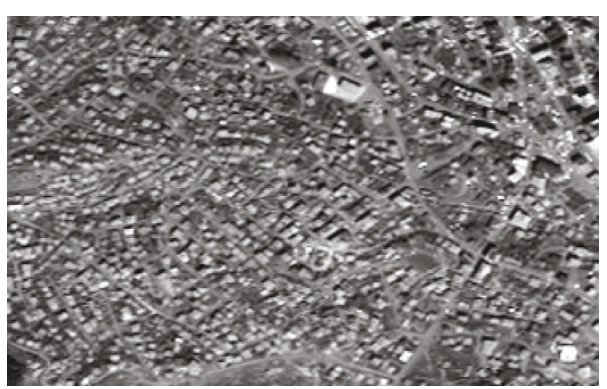

(b)

FIGURE 1: Bingol, Turkey area: (a) before the earthquake 2002-07-15; (b) after the earthquake 2003-05-02. (Digital Globe.)

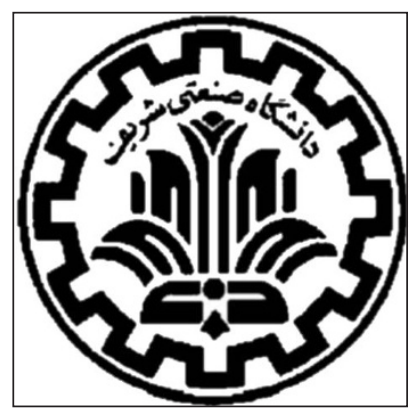

Figure 2: A sample image.

the pixels with cyan colors resulting from the borders of the transformed images are not because of any inaccuracy in the proposed registration method, but are caused by the lack of input data.

The rest of this paper is organized as follows. Section 2 describes the proposed method containing a discussion about the direct linear transform, the estimated affine transform, the related experimental results, and a proposed method to estimate the changes that have occurred on images. Section 3 contains the experimental results and discussions, and finally Section 4 concludes the paper. 


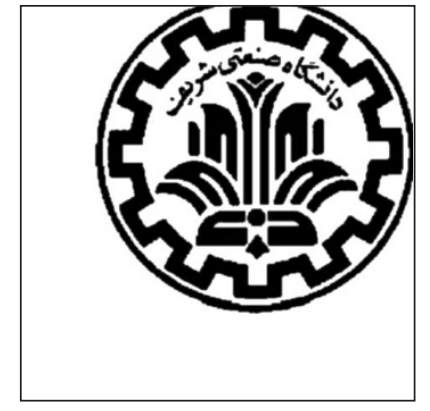

(a)

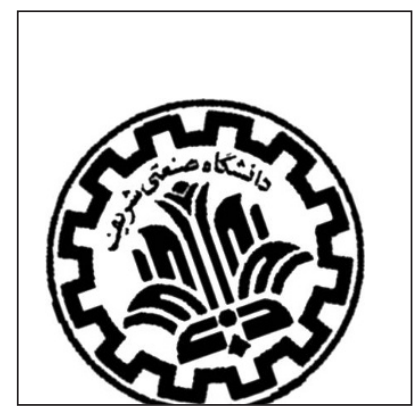

(b)

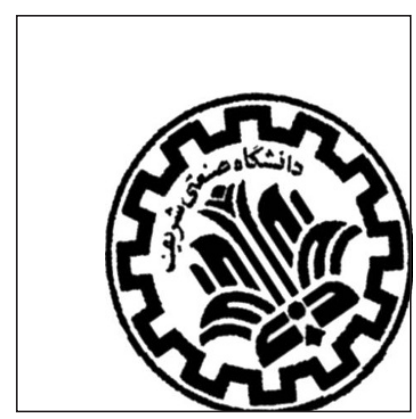

(c)

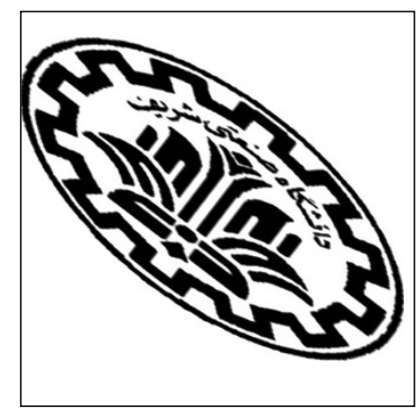

(d)

FIGURE 3: Different transformations of the logo image shown in Figure 2: (a) translated; (b) rotated and translated; (c) rotated, translated, and balanced scaled; (d) rotated, translated, and unbalanced scaled.

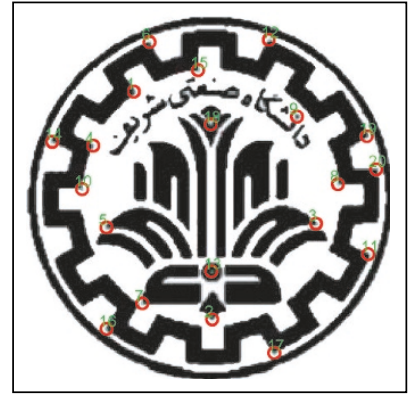

Figure 4: Control points overlaid on the logo image shown in Figure 2.

\section{PROPOSED METHOD}

Let images $I_{1}$ and $I_{2}$ correspond to two different captures of the same scene in different times. The aim of the registration stage is to find the transform $T:[x, y] \rightarrow\left[x^{\star}, y^{\star}\right]$ in the way that when applying the transform $T$ with the image $I_{2}$, the resulting image $I_{2}^{\prime}$ gets aligned with the image $I_{1}$. We call the control points in the two images of $I_{1}$ and $I_{2}$ as $\vec{x}_{i}$ and $\vec{y}_{i}$ for $i=1 \cdots n$, respectively. They are chosen so that applying the transform $T$ on $\vec{x}_{i}$, the result lies on $\vec{y}_{i}$. In fact, $\vec{x}_{i}$ and $\vec{y}_{i}$ correspond to the same physical location captured as an image pixel. Here, we assume that the used control points are properly distributed all over the images.

\subsection{Direct linear transform and affine transform}

Registration has a structural relation to the problem of camera calibration [21], where one is concerned with estimating the $3 \mathrm{D}$ coordinates of a point from its corresponding $2 \mathrm{D}$ coordinates in (at least) two different cameras. A well-known model for camera projection is the direct linear transform (DLT) by Abdel-Aziz and Karara [22]. Modeling a camera with 11 parameters, the DLT is able to compensate perspective distortions [22].

In the methodology of the DLT, each camera is modeled by 11 parameters and the projection of the point $\vec{p}_{a}=$ $\left[x_{a}, y_{a}, z_{a}\right]$ on a camera is defined as [22]

$$
\begin{aligned}
x_{b} & =\frac{a_{u} x_{a}+b_{u} y_{a}+c_{u} z_{a}+d_{u}}{a x_{a}+b y_{a}+c z_{a}+1}, \\
y_{b} & =\frac{a_{v} x_{a}+b_{v} y_{a}+c_{v} z_{a}+d_{v}}{a x_{a}+b y_{a}+c z_{a}+1} .
\end{aligned}
$$

Here, the denominator term $(\lambda=a x+b y+c z+1)$ applies the effects of the destination from $\vec{p}$ to the center of the camera on the projected point coordinates [22]. In the case of spaceborn imagery, there are two simplifications to be applied on the DLT formulation. Firstly, the vertical distance between the camera and the subject points, $z$, is assumed to be constant (because the camera plane is almost parallel to the subject [9]). Secondly, as the normal vector of the cameraplane 


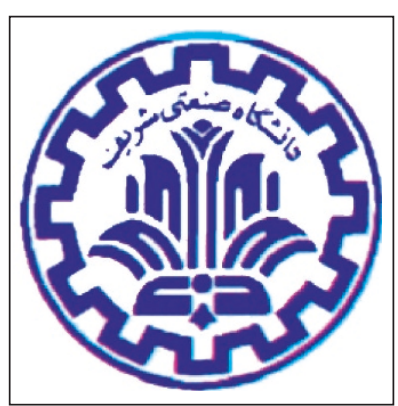

(a)

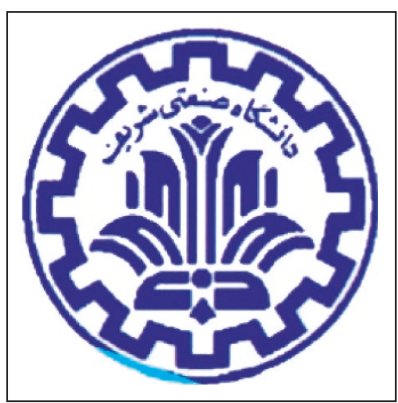

(b)

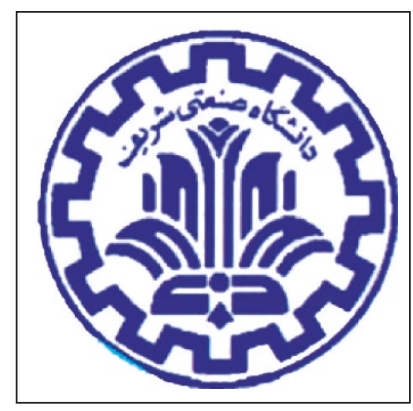

(c)

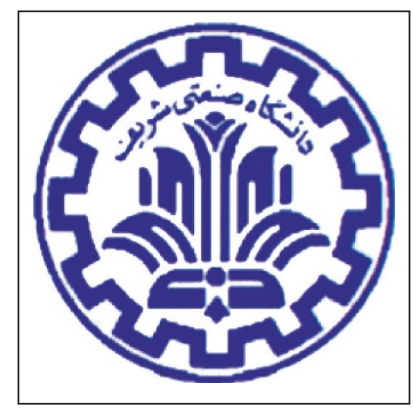

(d)

FIGURE 5: Results of performing the proposed estimated affine transform on the transformed images shown in Figure 3. and the normal vector of the "on the earth's" surface are almost parallel, the denominator term, $\lambda$, gets constant for all image pixels. Thus, setting

$$
\begin{aligned}
& a_{1}=\frac{1}{\lambda} a_{u}, \quad a_{2}=\frac{1}{\lambda} b_{u}, \quad t_{x}=\frac{1}{\lambda}\left(c_{u} z+d\right), \\
& a_{3}=\frac{1}{\lambda} a_{v}, \quad a_{4}=\frac{1}{\lambda} b_{v}, \quad t_{y}=\frac{1}{\lambda}\left(c_{v} z+d\right)
\end{aligned}
$$

gives the simplified linear model of

$$
\begin{aligned}
& x_{b}=a_{1} x_{a}+a_{2} y_{a}+t_{x}, \\
& y_{b}=a_{3} x_{a}+a_{4} y_{a}+t_{y},
\end{aligned}
$$

also known as the affine transform [9]. The affine transform can be written in the matrix notation as

$$
\vec{p}_{b}=\left(\begin{array}{ll}
a_{1} & a_{2} \\
a_{3} & a_{4}
\end{array}\right) \vec{p}_{a}+\left(\begin{array}{l}
t_{x} \\
t_{y}
\end{array}\right)
$$

Note that in contrast to the conventional DLT, here the two different parts of the affine transform (that result in determining the $x_{b}$ and $y_{b}$ parameters) can be solved independently resulting in fastening the algorithm efficiently.

The proposed algorithm for estimating the affine transform from CPs is based on the least-square error minimization approach.

\section{(1) Least-square method}

The quality of an affine transform can be measured by Err = $\sum_{i=1}^{N}\left\|\tilde{p}_{b, i}-p_{b, i}\right\|^{2}$. To minimize the transformation error, we have to set $\nabla$ Err $=0$ as

$$
\left(\begin{array}{l}
\frac{\partial \operatorname{Err}}{\partial a_{1}} \\
\frac{\partial E r r}{\partial a_{2}} \\
\frac{\partial \operatorname{Err}}{\partial a_{3}} \\
\frac{\partial \operatorname{Err}}{\partial a_{4}} \\
\frac{\partial \operatorname{Err}}{\partial t_{x}} \\
\frac{\partial \operatorname{Err}}{\partial t_{y}}
\end{array}\right)=\overrightarrow{0} .
$$


We can rewrite (8) as

$$
\begin{aligned}
a_{1} \sum_{i=1}^{N} x_{a, i}^{2}+a_{2} \sum_{i=1}^{N} x_{a, i} \cdot y_{a, i}+t_{x} \sum_{i=1}^{N} x_{a, i} & =\sum_{i=1}^{N} x_{b, i} \cdot x_{a, i}, \\
a_{1} \sum_{i=1}^{N} x_{a, i} \cdot y_{a, i}+a_{2} \sum_{i=1}^{N} y_{a, i}^{2}+t_{x} \sum_{i=1}^{N} y_{a, i} & =\sum_{i=1}^{N} x_{b, i} \cdot y_{a, i}, \\
a_{1} \sum_{i=1}^{N} x_{a, i}+a_{2} \sum_{i=1}^{N} y_{a, i}+t_{x} \cdot N & =\sum_{i=1}^{N} x_{b, i}, \\
a_{3} \sum_{i=1}^{N} x_{a, i}^{2}+a_{4} \sum_{i=1}^{N} x_{a, i} \cdot y_{a, i}+t_{y} \sum_{i=1}^{N} x_{a, i} & =\sum_{i=1}^{N} y_{b, i} \cdot x_{a, i}, \\
a_{3} \sum_{i=1}^{N} x_{a, i} \cdot y_{a, i}+a_{4} \sum_{i=1}^{N} y_{a, i}^{2}+t_{y} \sum_{i=1}^{N} y_{a, i} & =\sum_{i=1}^{N} y_{b, i} \cdot y_{a, i}, \\
a_{3} \sum_{i=1}^{N} x_{a, i}+a_{4} \sum_{i=1}^{N} y_{a, i}+t_{y} \cdot N & =\sum_{i=1}^{N} y_{b, i} .
\end{aligned}
$$

Now, using this derivation, we just need to solve two linear equations of order three simultaneously. Note that the computational complexity order of the proposed algorithm has reduced to only $O(N)$ instead of conventional approach that is in order of $O\left(N^{3}\right)$.

\section{(2) Experimental results}

The performance of the proposed algorithm is analyzed in terms of its complexity and accuracy. To implement the algorithm, we have used Matlab 6.5 on a $1.7 \mathrm{GHz}$ Intel Pentium M computer with $512 \mathrm{MB}$ of RAM. The accuracy of different algorithms to approximate the affine transform between two sets of CPs and the related error caused during the processes are listed in Table 1. The error is calculated using

$$
\text { Error }=\frac{1}{N} \frac{1}{\sqrt{W^{2}+H^{2}}} \sum_{i=1}^{N}\left|\vec{p}_{b, i}-\left(A \vec{p}_{a, i}+\vec{t}\right)\right|
$$

where $w$ and $h$ denote the width and height of the image, respectively. Table 2 lists the computational cost when using different number of CPs. (The common number of CPs depends on the application but an appropriate value is a number between 20-30.)

As the registration step plays an important role in the overall performance of any change detection approach, and the remotely sensed images cannot well illustrate the accurate performance of the proposed registration algorithm, here we have used a sample image (the logo of our university) to better illustrate the accurate performance of the proposed registration method.

\subsection{Proposed change detection method}

In this section, we state our proposed unsupervised method for segmentation and change detection in multispectral remotely sensed image intervals using the proposed fuzzy principal component analysis-based clustering method. While the proposed method is faster than the available approaches
TABle 1: Performance of different algorithms.

\begin{tabular}{lccc}
\hline Algorithm & Run time & Error & Stability \\
\hline Gradient-descent [23] & $2700 \mathrm{~ms}$ & $18.96 \%$ & No \\
Geometric [23] & $10 \mathrm{~ms}$ & $1.07 \%$ & Yes \\
Enhanced geometric [23] & $16 \mathrm{~ms}$ & $0.045 \%$ & Yes \\
Fourier transform [24] & $3.8 \mathrm{~ms}$ & $0.027 \%$ & Yes \\
Proposed LMS & $0.5 \mathrm{~ms}$ & $0.010 \%$ & Yes \\
\hline
\end{tabular}

TABLE 2: Required run time when using different number of control points.

\begin{tabular}{l|cccc}
\hline Number of CPs & $N=10$ & $N=20$ & $N=100$ & $N=200$ \\
\hline $\begin{array}{l}\text { Fourier } \\
\text { transform [24] }\end{array}$ & $1.06 \mathrm{~ms}$ & $3.8 \mathrm{~ms}$ & $108.95 \mathrm{~ms}$ & $445 \mathrm{~ms}$ \\
Proposed LMS & $0.34 \mathrm{~ms}$ & $0.50 \mathrm{~ms}$ & $2.43 \mathrm{~ms}$ & $4.72 \mathrm{~ms}$ \\
\hline
\end{tabular}

reported in the literature, and depends on no predetermined parameters, it is also robust against illumination changes. To the best knowledge of the authors, the method introduced in this paper is the first fuzzy change detection process. Note that the proposed affine transform estimation and the proposed change detection methods can also be used in other applications such as video motion estimation.

The literature of multispectral segmentation is not so rich compared to the case of gray-scale segmentation methods. The first significant method for measuring the colorbased similarity between two images might be the color histogram intersection approach introduced by Swain and Ballard [25]. Although, the method is very simple, it gives a relatively reasonable performance with two main shortcomings: the lack of spatial information about the images, and dependency on imaging conditions (like the ambient illumination). Some other researchers try to use certain color spaces that they believed to be suitable for segmentation purposes. For example in [26], the authors use a geometrical measure in the color histogram to define the similarity between color pairs in the HLS color space. Although some good segmentation results in the HLS color space are reported [27], it is proved in various studies that none of the standard color spaces are outperforming the others (e.g., see [28, 29]), while the local principal component analysis (PCA) is proved to give dominantly better results $[29,30]$. In [31], the researchers process color components independently, neglecting the vector tendency of them. In [32], motion estimation is used for segmentation purposes. Here, we used all $m-D$ data in our proposed PCA-based clustering and change detection stages.

Let two images $I_{1}$ and $I_{2}$ belong to the same scene. Then, each pixel in $I_{1}$ and $I_{2}$ is an $m-D$ realization. Also, let image $I_{1}$ be segmented into $c$ classes of $\phi_{i}$ using the proposed FPCAC method [33]. Here, $J_{i x y}$ shows the membership of $\vec{I}_{1 x y}$ to the ith class.

Now, perform the FPCA [33] on the fuzzy set,

$$
\tilde{X}=\left\{\left(\vec{I}_{2 x y} ; J_{i x y}^{m}\right) \mid 1 \leq x \leq W, 1 \leq y \leq H\right\},
$$

to find the new clusters $\widetilde{\phi}_{i}$. In fact, we are using the temporal redundancy of successive images, assuming that the fuzzy 
membership of a pixel to the $c$ classes remains constant if there is no abrupt change. The reason behind finding the new clusters in $I_{2}$ is to compensate probable slight changes corresponding to the lighting and sensor changes. Now, we have the new membership values $\widetilde{J}_{i x y}$, which show the level of membership of $\vec{I}_{2 x y}$ to the ith new class $\tilde{\phi}_{i}$.

We propose computing

$$
\delta_{x y}^{2}=\frac{1}{c^{2}} \sum_{i=1}^{c} J_{i x y}\left(J_{i x y}-\tilde{J}_{i x y}\right)^{2}, \quad 1 \leq x \leq W, 1 \leq y \leq H
$$

as the probability of the point $(x, y)$ being changed from $I_{1}$ to $I_{2}$. In fact, $\delta_{x y}$ measures the net amount of change in membership of pixels to the classes in the successive images. Note that while these fuzzy change values are computed, the clusters are also updated at the same time.

If $I_{1} \equiv I_{2}$, then $J_{i x y}$ and $\tilde{J}_{i x y}$ will be identical, resulting in $\delta_{x y}$ being zero everywhere, as desired. Now, assume that there is no change between the two images $I_{1}$ and $I_{2}$, unless for the changes in the imaging conditions. Assume that $\vec{x}_{i}$ and $\vec{y}_{i}$ are the spectral vectors of the same pixel in the two images $I_{1}$ and $I_{2}$, respectively. We model the change in imaging conditions as a linear operation [34]. Assume that $\vec{x}_{i}$ and $\vec{y}_{i}$ relate through a linear transform, namely, $\vec{x}_{i}=A \vec{y}_{i}+\vec{b}$. Here, we model $A$ as a nonsingular invertible matrix with its eigenvalues being almost constant. This situation relates to the cases that the spectral axes rotate (changing the chromaticity of the illumination), scale (changing the achromaticity of the illumination), and translate. The model restricts unbalanced scaling of spectral components which changes the spectral information non-meaningfully (for details see [34]). Note that matrix $A$ in the singular value decomposition (SVD) form is written as $A=V D U^{-1}$, where $U$ and $V$ are orthogonal matrices and $D$ is a diagonal matrix with the eigenvalues of $A$ as its elements.

The expectation vectors in the two images $I_{1}$ and $I_{2}$ relate as $E\left\{\vec{x}_{i}\right\}=E\left\{A \vec{y}_{i}+\vec{b}\right\}=A E\left\{\vec{y}_{i}\right\}+\vec{b}$. The fuzzy covariance matrices of the two images $I_{1}$ and $I_{2}$ satisfy $C_{1}=$ $A E\left\{\left(\vec{y}_{i}-E\left\{\vec{y}_{j}\right\}\right)\left(\vec{y}_{i}-E\left\{\vec{y}_{j}\right\}\right)^{T}\right\} A^{T}=A C_{2} A^{T}$. Assume that the eigenvectors of $C_{1}$ are $\vec{v}_{i}$ corresponding to the eigenvalues of $\lambda_{i}$ and the eigenvectors of $C_{2}$ are $\vec{u}_{i}$ corresponding to the eigenvalues of $\rho_{i}$. Also, assume the eigenvectors of $A$ to be $\vec{w}_{i}$ corresponding to the eigenvalues of $\varepsilon_{i}$. Thus, for all $i, C_{1} \vec{v}_{i}=\lambda_{i} \vec{v}_{i}, C_{2} \vec{u}_{i}=\rho_{i} \vec{u}_{i}$, and $A \vec{w}_{i}=\varepsilon_{i} \vec{w}_{i}$. First assume that the eigenvectors of $A$ are all exactly equal to the fixed value of $\lambda$ (or equivalently $\forall i, \varepsilon_{i}=\lambda$ ). Thus, $A=V D U^{-1}$ equals $V$ diagonal $(\lambda, \ldots, \lambda) U^{-1}=\lambda V U^{-1}$. In this situation, $A^{T}=\lambda U V^{-1}=\lambda^{2} A^{-1}$ resulting in $A^{T} A=A A^{T}=\lambda^{2} I$. Now, note that $C_{1} A \vec{u}_{i}=A C_{2} A^{T} A \vec{u}_{i}=\lambda^{2} A C_{2} \vec{u}_{i}=\lambda^{2} \rho_{i} A \vec{u}_{i}$. Thus, $A \vec{u}_{i}$ is the eigenvector of $C_{2}$ corresponding to the eigenvalue of $\lambda^{2} \rho_{i}$. Note that $\left\|A \vec{u}_{i}\right\|=\lambda\left\|\vec{u}_{i}\right\|=\lambda$. As the eigenvalues and eigenvectors of a single matrix are identical, $\left\{\left((1 / \lambda) A \vec{u}_{1}, \lambda^{2} \rho_{1}\right), \ldots,\left((1 / \lambda) A \vec{u}_{m}, \lambda^{2} \rho_{m}\right)\right\}$ is identical to $\left\{\left(\vec{v}_{1}, \lambda_{1}\right), \ldots,\left(\vec{v}_{m}, \lambda_{m}\right)\right\}$. As $\lambda^{2}>0$, we have $\vec{v}_{i}=(1 / \lambda) A \vec{u}_{i}$ and $\lambda_{i}=\lambda^{2} \rho_{i}$, for all $i$. Thus, using the above reclustering method, the cluster $\phi=[\vec{\eta}, \vec{v}]$ in $I_{2}$ results in the cluster

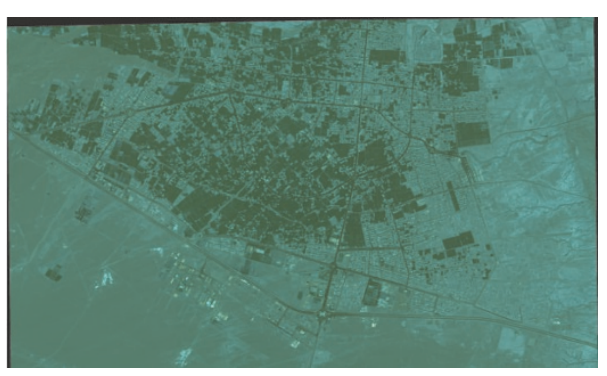

(a)

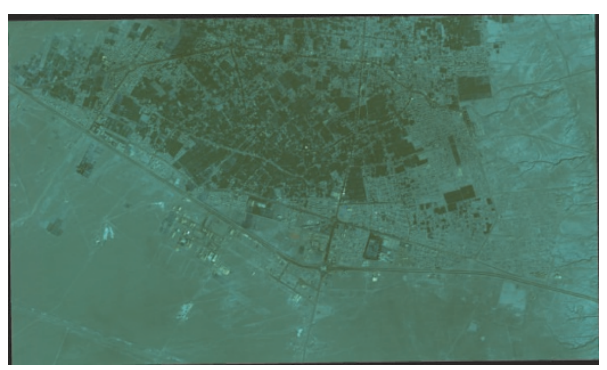

(b)

FIGURE 6: $\mathrm{Bam}$ area: (a) unregistered image before the earthquake 2003-12-04; (b) unregistered image after the earthquake 2003-1229. (Digital Globe.)

$\tilde{\phi}=[A \vec{\eta}+\vec{b}, A \vec{v}]$. Now, we have

$$
\begin{aligned}
\Psi\left(\vec{x}_{i}, \tilde{\phi}\right)= & \|\left[\left(A \vec{y}_{i}+\vec{b}\right)-(A \vec{\eta}+\vec{b})\right] \\
& \quad-\frac{1}{\lambda^{2}} \vec{v}^{T} A^{T}\left[\left(A \vec{y}_{i}+\vec{b}\right)-(A \vec{\eta}+\vec{b})\right] A \vec{v} \|^{2} \\
= & \lambda^{2} \Psi\left(\vec{x}_{i}, \tilde{\phi}\right),
\end{aligned}
$$

and $\tilde{J}_{i x y}=J_{i x y}$, resulting in $\delta_{x y}=0$. Thus, the proposed method will be independent of the lighting and imaging conditions. Now, assume a more realistic case that $\varepsilon_{i}$ 's are not exactly the same but we have $\lambda-\delta \lambda \leq \varepsilon_{i} \leq \lambda+\delta \lambda$. For the cases that $\delta \lambda / \lambda$ is too small, the above equations change to semiequations and still marginally hold. In this situation $\delta_{x y} \simeq 0$. In contrast, physical changes of interest result in different materials in a single point in different shots. Hence, they produce absolutely different values of $J_{i x y}$ and $\tilde{J}_{i x y}$ resulting in nonzero patterns of $\delta_{x y}$. In the proposed method, at the same time both the image sequence segmentation and the fuzzy change detection are performed.

\section{EXPERIMENTAL RESULT}

The experiments are performed using an Intel Centrino $1700 \mathrm{MHz}$ computer with $512 \mathrm{MB}$ of RAM. 


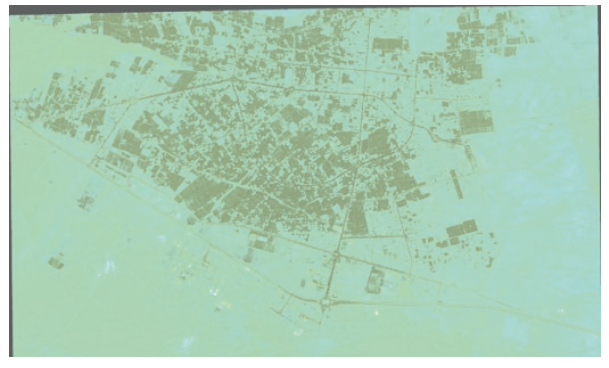

(a)

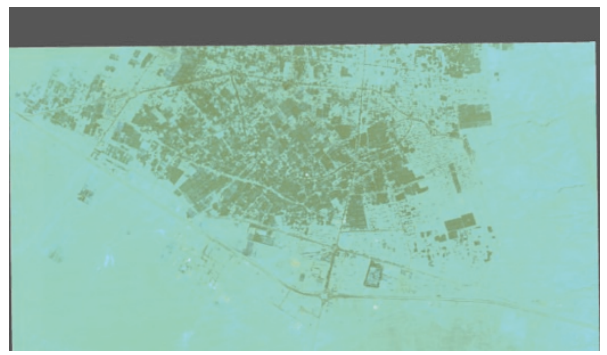

(b)

FIgURE 7: Bam area: (a) registered image before the earthquake 2003-12-04; (b) registered image after the earthquake 2003-12-29.

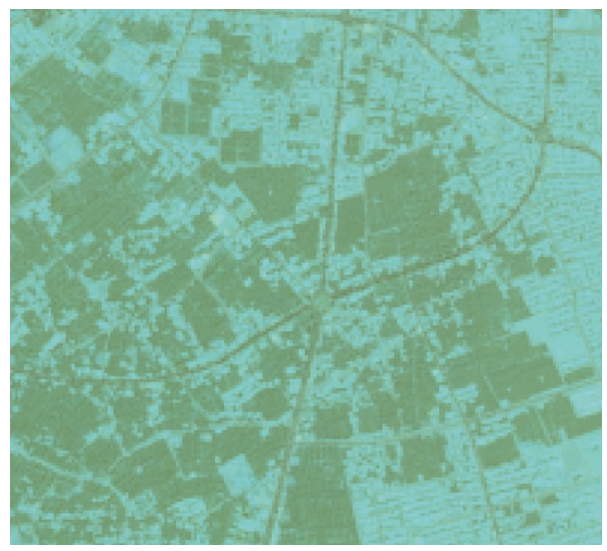

(a)

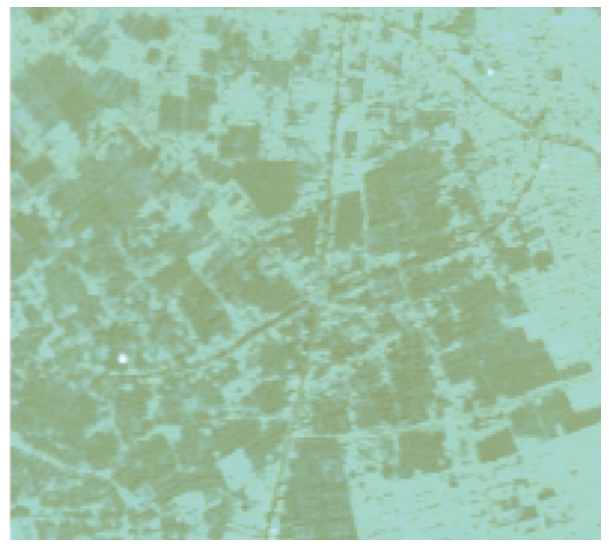

(b)

FIgURE 8: Urban portion of the images shown in Figure 7.

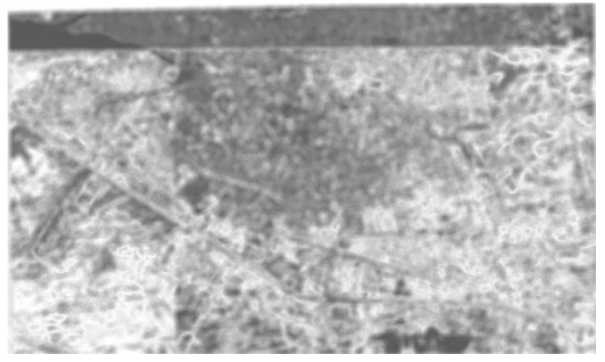

(a)

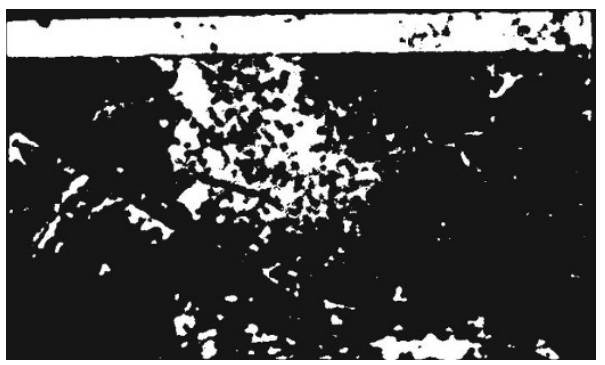

(b)

FIGURE 9: Resulting change maps using the proposed change detection algorithm: (a) fuzzy change map; (b) crisp change map (after hard thresholding).

Figure 6 shows two multiband images taken from the city of Bam by the Quick Bird satellite, before and after the devastating earthquake of December 26, 2003 before registration. Figure 7 shows the result of our registration. Figure 8 shows the urban portion of the images. The first images are cropped with no magnification to focus on details.

Figure 9 shows the resulted fuzzy change maps. A crisp map can be easily generated after performing a hard threshold.

As mentioned before, the proposed algorithm computes both the segmentation and the change detection map at the same time. Note that many applications need to use them at the same time. Figure 10 illustrates the segmentation result before the earthquake and the segmentation tuning result after the earthquake.

To show the robustness of the proposed algorithm against changes in imaging conditions, we have evaluated its change detection performance when running it on two images with manipulated color changes. In fact, Figure 11 shows a simulated change in imaging conditions with no real changes on the earth's surface. Figures 12 and 13 illustrate the robustness of the proposed algorithm against such changes. Here, we chose a linear transform with eigenvalues 0.9, 0.7, 0.9, which are not completely equal to simulate the more realistic changes. When running the proposed change detection stage on $472 \times 792$ downsampled images, it elapsed $5.7 \mathrm{sec}-$ onds.

\section{CONCLUSION}

In this paper, a fast and accurate affine transform estimation method and a new efficient fuzzy change detection method are proposed for remotely sensed images. The 


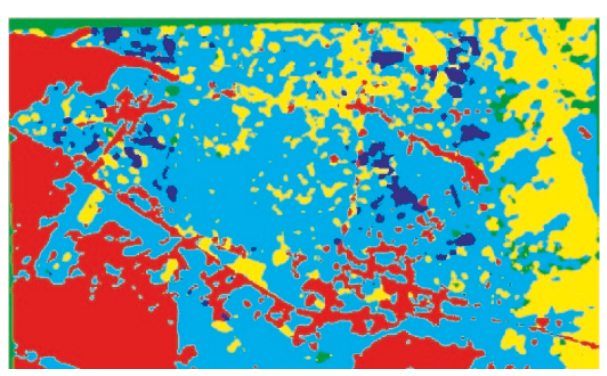

(a)

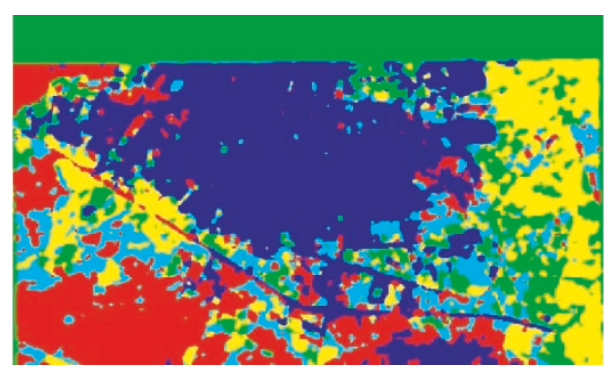

(b)

Figure 10: Segmentation results: (a) before the earthquake; (b) segmentation tuning after the earthquake.

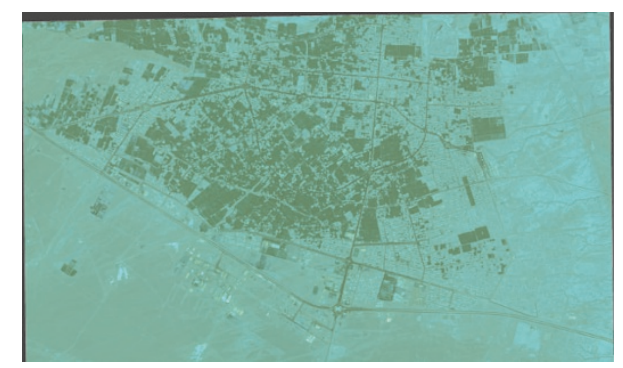

FIGURE 11: Linearly changed image.

experimental results show that the proposed method is fast and robust against undesired change in imaging conditions. It was shown that the algorithm can be also efficiently used to detect damages caused by an earthquake.

\section{ACKNOWLEDGMENTS}

This work was in part supported by a Grant from ITRC. We would like to appreciate the valuable discussions and suggestions made by Professor M. Nakamura and Professor Y. Kosugi from Tokyo Institute of Technology. We also wish to thank the Iranian Remote Sensing Center (IRSC) and Digital Globe for providing us with the remotely sensed images used in this paper. Arash Abadpour also wishes to thank Ms. Azadeh Yadollahi for her encouragement and invaluable ideas.

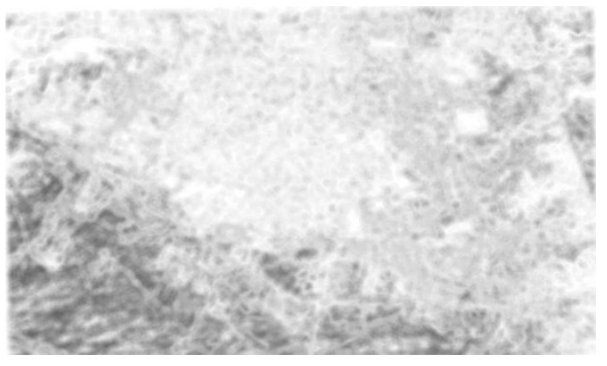

(a)

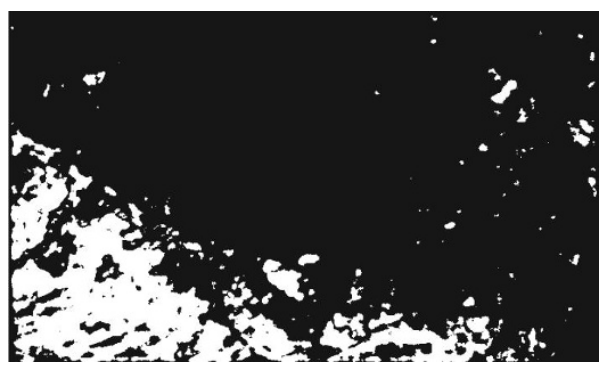

(b)

FIGURE 12: Resulting change maps using the proposed change detection method (linearly changed image): (a) fuzzy change map; (b) crisp change map (after hard thresholding).

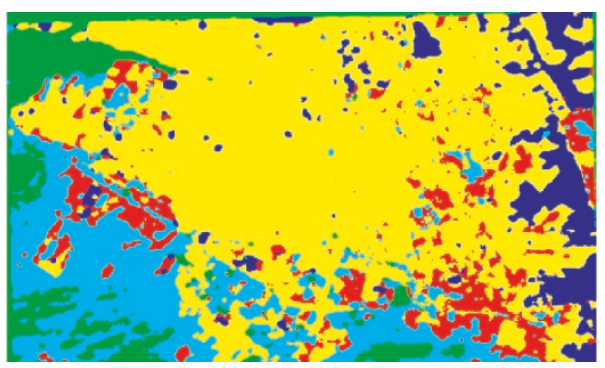

(a)

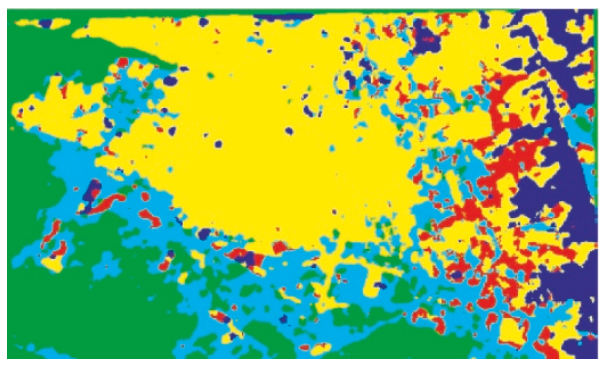

(b)

FIGURE 13: Segmentation results: (a) original image; (b) linearly changed image.

\section{REFERENCES}

[1] R. Wiemker, A. Speck, D. Kulbach, H. Spitzer, and J. Bienlein, "Unsupervised robust change detection on multispectral imagery using spectral and spatial features," in Proceedings of the 3rd International Airborne Remote Sensing Conference and 
Exhibition, vol. 1, pp. 640-647, Copenhagen, Denmark, July 1997.

[2] C. S. Fischer and L. M. Levien, "Monitoring California's hardwood rangelands using remotely sensed data," in Proceedings of the 5th Symposium on Oak Woodlands, San Diego, Calif, USA, October 2001.

[3] K. M. Bergen, D. G. Brown, J. R. Rutherford, and E. J. Gustafson, "Development of a method for remote sensing of land-cover change 1980-2000 in the USFS North Central Region using heterogeneous USGS LUDA and NOAA AVHRR 1 $\mathrm{km}$ data," in Proceedings of IEEE International Geoscience and Remote Sensing Symposium (IGARSS '02), vol. 2, pp. 12101212, Toronto, Ontario, Canada, June 2002.

[4] M. Matsuoka and F. Yamazaki, "Application of the damage detection method using SAR intensity images to recent earthquakes," in Proceedings of IEEE International Geoscience and Remote Sensing Symposium (IGARSS '02), vol. 4, pp. 20422044, Toronto, Ontario, Canada, June 2002.

[5] G. Andre, L. Chiroiu, C. Mering, and F. Chopin, "Building destruction and damage assessment after earthquake using high resolution optical sensors. The case of the Gujarat earthquake of January 26, 2001," in Proceedings of IEEE International Geoscience and Remote Sensing Symposium (IGARSS '03), vol. 4, pp. 2398-2400, Toulouse, France, July 2003.

[6] M. Nakamura, M. Sakamoto, S. Kakumoto, and Y. Kosugi, "Stabilizing the accuracy of change detection from geographic images by multi-leveled exploration and selective smoothing," in Proceedings of the Global Conference and Exhibition on Geospatial Technology, Tools and Solutions (GIS '03), Vancouver, BC, Canada, March 2003.

[7] L. G. Brown, "A survey of image registration techniques," ACM Computing Surveys, vol. 24, no. 4, pp. 325-376, 1992.

[8] J. R. G. Townshend, C. O. Justice, C. Gurney, and J. McManus, "The impact of misregistration on change detection," IEEE Transactions on Geoscience and Remote Sensing, vol. 30, no. 5, pp. 1054-1060, 1992.

[9] B. Zitova and J. Flusser, "Image registration methods: a survey," Image and Vision Computing, vol. 21, no. 11, pp. 9771000, 2003.

[10] D. Robinson and P. Milanfar, "Fundamental performance limits in image registration," in Proceedings of IEEE International Conference on Image Processing (ICIP '03), vol. 3, pp. 323-326, Barcelona, Spain, September 2003.

[11] H. Li , B. S. Manjunath, and S. K. Mitra, "A contour-based approach to multisensor image registration," IEEE Transactions on Image Processing, vol. 4, no. 3, pp. 320-334, 1995.

[12] M. Xia and B. Liu, "Image registration by "Super-curves", IEEE Transactions on Image Processing, vol. 13, no. 5, pp. 720732, 2004.

[13] M. Helm, "Towards automatic rectification of satellite images using feature based matching," in Proceedings of International Geoscience and Remote Sensing Symposium (IGARSS '91), vol. 4, pp. 2439-2442, Espoo, Finland, June 1991.

[14] A. Goshtasby and G. C. Stockman, "Point pattern matching using convex hull edges," IEEE Transactions on Systems, Man and Cybernetics, vol. 15, no. 5, pp. 631-637, 1985.

[15] Y. C. Hsieh, D. M. McKeown, and F. P. Perlant, "Performance evaluation of scene registration and stereo matching for cartographic feature extraction," IEEE Transactions on Pattern Analysis and Machine Intelligence, vol. 14, no. 2, pp. 214-238, 1992.

[16] M. Roux, "Automatic registration of SPOT images and digitized maps," in Proceedings of IEEE International Conference on Image Processing (ICIP '96), vol. 2, pp. 625-628, Lausanne, Switzerland, September 1996.

[17] S. Z. Li, J. Kittler, and M. Petrou, "Matching and recognition of road networks from aerial images," in Proceedings of the 2nd European Conference on Computer Vision (ECCV'92), pp. 857-861, Santa Margherita Ligure, Italy, May 1992.

[18] M. Sester, H. Hild, and D. Fritsch, "Definition of groundcontrol features for image registration using GIS-data," in Proceedings of the Symposium on Object Recognition and Scene Classification from Multispectral and Multisensor Pixels CDROM, vol. 32/3, pp. 537-543, Columbus, Ohio, USA, 1998.

[19] H. Maître and Y. Wu, "Improving dynamic programming to solve image registration," Pattern Recognition, vol. 20, no. 4, pp. 443-462, 1987.

[20] H. Neemuchwala, A. Hero, and P. Carson, "Image registration using alpha-entropy measures and entropic graphs," European Journal on Signal Processing, 2004, Special issue on contentbased visual information retrieval, http://www.elsevier.nl/ locate/sigpro.

[21] C. Chatterjee and V. P. Roychowdhury, "Algorithms for coplanar camera calibration," Machine Vision and Applications, vol. 12, no. 2, pp. 84-97, 2000.

[22] Y. Abdel-Aziz and H. Karara, "Direct linear transformation from comparator coordinates into object space coordinates in close range photogrammetry," in Proceedings of the ASP/UI Symposium on Close-Range Photogrammetry, pp. 1-18, Urbana, Ill, USA, January 1971.

[23] A. Abadpour and S. Kasaei, "Fast registration of remotely sensed images," in Proceedings of the 10th Annual Computer Society of Iran Computer Conference (CSICC '05), pp. 61-67, Tehran, Iran, February 2005.

[24] S. M. Amiri and S. Kasaei, "An ultra fast method to approximate affine transform using control points," submitted to IEEE Signal Processing Letters.

[25] M. J. Swain and D. H. Ballard, "Color indexing," International Journal of Computer Vision, vol. 7, no. 1, pp. 11-32,1991.

[26] H. Y. Lee, H. K. Lee, and Y. H. Ha, "Spatial color descriptor for image retrieval and video segmentation," IEEE Transactions on Multimedia, vol. 5, no. 3, pp. 358-367, 2003.

[27] D. Androutsos, K. Plataniotis, and A. Venetanopulos, "Efficient indexing and retrieval of color image data using a vectorbased approach," Ph.D. dissertation, University of Toronto, Toronto, Ontario, Canda, 1999.

[28] M. C. Shin, K. I. Chang, and L. V. Tsap, "Does colorspace transformation make any difference on skin detection?" in Proceedings of the 6th IEEE Workshop on Applications of Computer Vision (WACV'02), pp. 275-279, Orlando, Fla, USA, December 2002.

[29] A. Abadpour and S. Kasaei, "A new parametric linear adaptive color space and its PCA-based implementation," in Proceedings of the 9th Annual Computer Society of Iran Computer Conference (CSICC '04), vol. 2, pp. 125-132, Tehran, Iran, February 2004.

[30] A. Abadpour and S. Kasaei, "Performance analysis of three homogeneity criteria for color image processing," in Proceedings of IPM Workshop on Computer Vision, Tehran, Iran, April 2004.

[31] Y. Du, C.-I. Chang, and P. D. Thouin, "Unsupervised approach to color video thresholding," Optical Engineering, vol. 43, no. 2, pp. 282-289, 2004.

[32] I. Patras, E. A. Hendriks, and R. L. Lagendijk, "Semi-automatic object-based video segmentation with labeling of color segments," Signal Processing: Image Communication, vol. 18, no. 1, pp. 51-65, 2003. 
[33] A. Abadpour and S. Kasaei, "A new FPCA-based fast segmentation method for color images," in Proceedings of the 4th IEEE International Symposium on Signal Processing and Information Technology (ISSPIT '04), pp. 72-75, Rome, Italy, December 2004.

[34] D. P. Nikolaev and P. P. Nikolayev, "Linear color segmentation and its implementation," Computer Vision and Image Understanding, vol. 94, no. 1-3, pp. 115-139, 2004.

Arash Abadpour received his B.S. degree from Control Group, Department of Electrical Engineering, Sharif University of Technology (SUT), Tehran, Iran, in 2003. He is currently a master's student in Computer Science Group, Department of Mathematical Science, Sharif University of Technology, Tehran, Iran. His research interests are in image processing with primary emphasis on color image processing.

Shohreh Kasaei received her B.S. degree from Department of Electronics, Faculty of Electrical and Computer Engineering, Isfahan University of Technology (IUT), Iran, in 1986. She worked as a Research Assistant in Amirkabir University of Technology (AUT) for three years. She then received her M.S. degree from Graduate School of Engineering, Department of Electrical and Electronic Engineering, University of the Ryukyus, Japan, in 1994, and her Ph.D. degree at Signal Processing Research Centre (SPRC), School of Electrical and Electronic Systems Engineering (EESE), Queensland University of Technology (QUT), Australia, in 1998. She was awarded as the best graduate student in engineering faculties of University of the Ryukyus, in 1994, the best Ph.D. Students Studied in Overseas by the Ministry of Science, Research, and Technology of Iran, in 1998, and as a Distinguished Researcher of Sharif University of Technology (SUT), in 2002, where she is currently an Associate Professor. Her research interests are in image processing with primary emphasis on object-based video compression, content-based image retrieval, video restoration, motion estimation, virtual studios, fingerprint authentication $\backslash$ identification, tracking, color $\backslash$ multispectral image processing, and multidimensional signal modeling and prediction. Also, multiscale analysis with application to image \video compression, image enhancement, pattern recognition, motion tracking, texture segmentation and classification, and digital video watermarking.

S. Mohsen Amiri received his B.S. degree from Department of Electronics, Faculty of Electrical and Computer Engineering, Isfahan University of Technology (IUT), Iran, in 2004. He worked as a Research Assistant in IUT, AI-Lab from 2002 to 2003 . He joined IUT Robotic-Center in 2003 and was awarded the 3rd place in Robocup World Cup, Italy, in 2003. He is currently a master's student in Artificial Intelligence Group, Department of Computer Engineering, Sharif University of Technology (SUT), Tehran, Iran. His research interests are in signal and

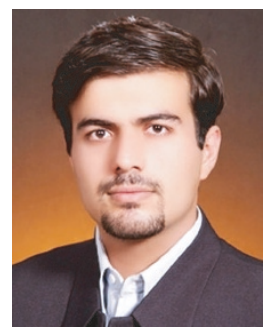

image processing with primary emphasis on color $\backslash$ multispectral image processing and multidimensional texture segmentation and classification, data mining, algorithm design, and optimization systems. 